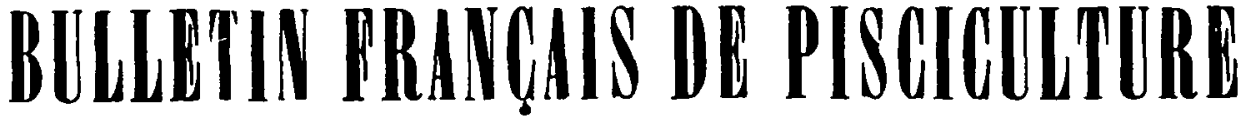

Première AnNée

No 11

MAI 1929

SOMMAIRE. - SABatier de LaChadenède : La conservation des Poissons migrateurs et l'aménagement hydraulique des cours d'eau. - L. Bousset et.J. LARRisu : Quelques expériences sur la reproduction du Saumon. - Expériences de génétique. - C. Gươ : Jurisprudence. - Chronique : Quel sort faut-il réserver à la Truite arc-en-ciel ? - Mise en valeur des réservoirs artificiels hydrauliques. - La Pisciculture et l'élevage de la ('arpe en Bohême. - Comminications conporatives : Cnion nationale des Syndicats de l'étang. - Syndicat des pisciculteurs-salmoniculteurs de France. - Syndicat des étangs de la Marne. - Syndicat des étangs de la Meuse. - Syndicat d'aquiculture du Centre. - Syndicat des étangs de Sologne. - Syndicat des étangs du Forez. - Chambre syndicale des étaugs de Touraine et d'Injou. - Consultations technques. - Renseignements commerciaux.

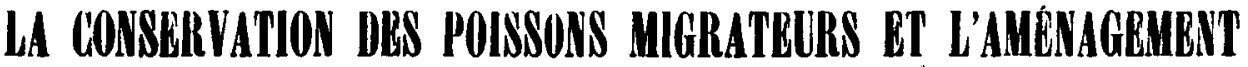 HYPRAULLOUR DES COUBS I"BAU}

\author{
Par M. SABATIER dE LACHADENEDEE \\ Conservatiur des Eaux et Forèts.
}

L'aménagement hydraulique des cours d'eau prend, en France, une extension de plus en plus considérable. C'est ainsi que, dans les plaines, on construit des barrages de dérivation el, dans les régions montagneuses, des barrages d'accumulation ; partout on coupe les rivières et on empêche la libre circulation du Poisson.

Les résultats néfastes se font surtout sentir quand il s'agit de migrateurs dont le Saumon représente le type le plus précieux.

Nous ne citerons pas les noms de tous les barrages dont la construction a, subitement, fait disparaitre le Saumon des cours d'eau. Cependant nous indiquerons que le Gave de Pau, autrefois poissonneux, ne renferme plus, aujourd'hui, aucun Saumon et, cela, depuis la construction du barrage de Castetarbe ; le Gave de Mauléon, où le Saumon n'avait plus accès depuis la construction du barrage d'Osserain, est fréquenté, à nouveau, depuis rg19, date de la rupture de cet ouvrage.

Dans la Nive, le Saumon remonte jusqu à Saint-Jean-Pied-de-Port et est arrêté à ce point par le barrage de mòme nom.

Les exemples pourraient ainsi se multiplier à I'infini.

Les dommages causés sont considérables, surtout quand il s'agit du Saumon dont le prix atteint facilement so francs le kilo.

Ils le sont encore plus si l'on considère, qu'outre le Saumon, l'Anguille et l'Alose sont des Poissons qui viennent de la mer pour remonter nos cours d'eau et en constituer une importante richesse.

Enfin, la libre circulation du Poisson indigène est entravée et celte entrave le gêne considérablement dans les actes de sa reproduction.

On ne saurait donc nier, qu'au tolal, le découpage de nos cours d'eau en biefs n'ayant, au point de vue piscicole, aucune communication entre eux, est un fạit très regrettable pour l'avenir du Poisson. 
Le Ministère de l'Agriculture s'ent est très justenent énu et a recherché les moyens propres à remédier à un tel état dé choses.

On a, jusqu à ce jour, préconisé les échelles à Poissons dont de nombreux systèmes ont été inventés. Yous ne pouvons, dans le cadre très réduit de cette notice, les décrire tous.

Nous citerons seulement l'échelle Denil dont certains font le plus grand éloge et l'échelle en bassins sur lesquels les suisses et les Allemands fondent les plus grands espoirs.

L'échelle Denil est trop connue pour que nous en parlions.

Léchelle en bassins avec échancrures est moins vulgarisée. Nous demandons la permission de la décrire succinctement.

Elle est formée de bassins superposés ayant, chacun, $1^{\text {m }}$ jo de longueur, $\mathrm{I}^{\mathrm{m}} 30$ de largeur et $\mathrm{I}$ mètre de profondeur.

Chacun de ces bassins a une échancrure de $o^{\text {mil }}$ ho de largeur sur $o^{\mathrm{m}} 80$ de hauteur.

La différence du niveau (l'eau entre chaque bassin doit être de $o^{\mathrm{m}} 20$ à $o^{\text {ma }} 25$. Le débit de cette échelle est de 5 à $6 u c$ litres-seconde.

Les échancrures ne sont pas exactement sur une ligne passant par l'axe de chaque bassin. Elles sont déportées légèrement et progressivement soit à droite, soit à gauche.

Celte échelle donne d'excellent résultats et paraît devoir être généralisée en Suisse et en Allemagne.

Elle serait, paraît-il, supérieure à l'échelle Denil, car celle-ci annule bien la vitesse de I'eau et réduirait ainsi, du moins théoriquement, I'effort que le Poisson a à laire. Mais chacun de ses eléments crée un remous, de telle sorte qu'il n'y a, dans l'eau descendante, aucun filet liquide en ligne droite.

Or, les Poissons poussés par leur instinct et aussi, peut-être, par le sens spécial des organes qu ils ont dans la ligne latérale, remontent un courant quand ils sentent la direction bien nette des filets liquides de ce courant. Ils se trouvent désorientés, ils sont affolés, ils sont perdus, dans des tourbillons oì ces filets liquides se croisent et s'entrecroisent, sans direction unique.

L'échelle en bassins, ne présentant pas l’inconvénient du remous, serait donc supérieure à l'échelle Denil.

Quoi qu'il en soit, l'échelle ne peut pas être pratiquement établie quand il s'agit de franchir une hauteur de barrage de plus de 6 à 7 mètres environ.

Chaque bassin faisant gagner $0^{\mathrm{m}} 25$ de différence de niveau, pour franchir 7 mètres de hauteur, il faudra 28 bassins. L'échelle devra donc avoir 3. mètres de longueur et il semble bien que ce soit là un maximum que l'on ne peut dépasser.

Celle échelle pourra, d'ailleurs, ne pas âtre rectiligne si les dispositions locales l'exigent. Il faudra, seulement, que son pied soit bien placé pour que les Poissons le trouvent facilement. 
Pour les barrages jusqu`à ; mètres de hauteur environ, avec un débit de 5 à 600 litres-seconde, nous avons un moyen d'établir une communication entre le bief aval et le bief amont.

Mais là se bornent nos possibilités. Si le barrage est plus élevé ou si le débit du cours d'eau n'est pas suffisant pour sacrifier is à boo litres-seconde, nous sommes sans moyen d'action.

Nous sommes, encore, sans moyens d'action quand la dérivation, absorbant tout le débit du cours d'eau, laisse, en aval du barrag̣e, une partie plus ou moins considérable de cours d'eau à sec.

Voici cependant une solution au problème, solution qui est venue à l'esprit inventif et avisé de notre camarade et ami, M. l'Inspecteur principal des Faux et Forêts ne Fouvir...e.

Pourquoi n'installerait-on pas, en aval du barrage ou du point d'évacuation des eaux des turbines, un système de pêcherie connu sous le nom de baro ou filet tournant?

Ce filet, actionné par l'cau. pêcherait d'une manière continue tout ce qui se présenterait à la remontée et déverserait le produit de sa pêche sur un trottoir roulant actionné par un petil moteur électrique.

Ce trottoir roulant, matelassé pour que le Poisson ne se blesse pas, fermé pour qu'il ne saute pas dans la rivière, conduirait celui-ci vers le bief amont où il le déverserait.

Naturellement, il y aurait lieu de prendre quelques précautions pour amener le Poisson vers le filet, pour empècher les crues de détruire l'installation. Mais l'idée, en elle-même, est des plus heureuses et facilement. réalisable.

Dès lors, plus de perte d'eau pour faire marcher l'échelle, plus d'obstacle quelle que soit la hauteur du barrage.

Pour le cas où le lit du cours d'eau serait à sec en aval du barrage, la pécherie serait installée dans le voisinage de la sortie des turbines et le poisson serait remonté jusqu'à un petit canal de niveau avec le bief amont. Dans ce cas seulement, il faudrait prélever sur le débit du cours d'eau, le débit de ce canal qui pourrait être d'ailleurs de peu d'importance.

L'idée mérite d'être expérimentée et sa rénssite solutionnerait, d'une manière absolument parfaite, la question de la conservation du peuplement piscicole des Poissons migrateurs.

\section{NOTE}

J'ai beaucoup d'obligations a mon excellent camarade M. le Conserviteur nf Lachavexìde, qui vcut bien juger diogne d'intérêt la substitution, a "l'échelle ", toujours coûteuse et trop souvent inefficace, de l' "ascenseur à Poissons". Jusqu'ici, en effet, cette innovation, que j'ai soumise à l'Administration, voici tantòt neuf ans, a paru trop lépourvue de préredents pour être mise à l'essai.

Pourtant le baro navarrais et basquais a la réputation d'òtre un engin efficace pour la pêche des Potamotoques en instance de remonte; il a été spécialcinent imaginé ct disposé à cet effet. Vers la fin du siècle dernier on admettait qu'un de ces filets tourmants capturait bon an, mal an, sur le Gave d'Oloron, 150 ì 200 Sammons adultes, ciant interdil de diriger les Poissons vers lui au moven de couloirs, ce qui ent accru 
sensiblement le rendement. Depuis la guerre les sportifs ont mené campagne si ircharnce contre les baros qu'ils ont abouti à les faire interdire comme destructeurs

Il paraîtrait donc indiqué d'utiliser pareil instrument à des fins, non plus balieuijques, mais piscicoles, en s'en servant pour cueillir, à proximité des barrages, les saumons dont le voyage nuptial risque d'être interrompu par ces obstacles. Ceci d'autant mieux que, grâce à un ensemble de dispositifs fort ingénieux, le baro fonc. tionne automatiquement et transfère ses prises de l'eau libre dans une bouticue ì l'oissons sans qu'ils aient ä subir d'atteinte brutale.

Pour le transformer en "ascenseur " il suffit de recevoir les animaux a la sortie du filet capteur, non plus sur une glissiere, mais sur un tapis roulant. Ce dernier les élèvera à la hauteur voulue et les déversera dans le hief d'amont. Sauf à prendre certaines dispositions particulières, le problème à résoudre n'est cerles par plus ardu que celui consistant à hisser du rez-de-chaussce à un étige quelconque lat clientèle des Magasins du Louvre.

Il se peut, naturellement, que des difficultés d'application surgissent. Mais commenl les connaître - et apprécier si elles sont insurmontables - si on ne fait des essais :

Sans doute, ces derniers exigeront-ils des frais... Reculera-t-on devant cux alor qu'on a prodigué et prodigue encore l'argent pour des échelles qui, depuis tani ¿lannées, ont donné mainte el mainte déception? Et ne faut-il pas tenir compte, aussi, de l'écónomie considérable à réaliser, avec le " baro ascenseur " même si son efficaciti: ne so révélait pas supérieure à celle des passes Caméré, Denil et aulres ? Bovvircr.

\title{
QUELQUES EXPÉRIENCES SUR LA REPRODUCTION DU SAUMON \\ (SALMO SALAR, L)
}

\author{
Par M. le Docteur Lovis BOUISSET
}

Chef des travaux à l'Institut d'Hydrobiologie et de Pisciculture de Toulouse, Assistant à la Faculté des Sciences.

et M. JEAN LARRIEL

Inspecleur des Laux et Forìts, à Oloron.

Il est généralement admis que le Saumon, avant de se reproduire, doit séjourner plus ou moins longtemps à la mer ; c’est pendant ce séjour qu'il effectue sa principale croissance. Ensuite, devenu adulte, il revient frayer en rivière.

Si cela paraît exact pour les femelles, les màles, - tout au moins dans le bassin de l'Adour (Nives et laves), - sont sexuellement mûrs deux ans après leur naissance, atant d'avoir jamais été à la mer, ainsi que l'a démontré l'un de nous (I-)). Leur laitance est fonctionnelle et l'on sait que des bandes de Tocans hantent les frayères au moment de la ponte des femelles. Leur présence, dit-on parfois, s'explique par leur voracité :

(1) Bourssfr (Louis). - Maturité sexuelle pricoce du Salumor (Salmo salar). C. R. H. Société de Biologie, r'́́ janv, 1928. XCVIII. 92.

(2) Il est vrai de dire que I'on a signalé le fait pour "quelques alevins males attardís en eau douce ". En nous basant sur l'étude des écailles de Saumons revenant de la mer, ou de Torans, nous pouvous affimer que ce ne sont pas des alcvins attardés qui sont mûrs dans nos régrions, mais bien des alevins de 2 ans qui constituent les $70 \%$ du contingent annuel de descente. 